\title{
Skin Lesion Classification Based on Deep Convolutional Neural Networks Architectures
}

\author{
Jwan Najeeb Saeed $^{1 *}$, Subhi R. M. Zeebaree ${ }^{2}$ \\ ${ }^{1}$ IT Department, Duhok Technical Institute, Duhok Polytechnic University, Duhok, Kurdistan Region, Iraq, \\ jwan.najeeb@dpu.edu.krd \\ ${ }^{2}$ Duhok Polytechnic University, Duhok, Iraq, subhi.rafeeq@dpu.edu.krd \\ Correspomdance: jwan.najeeb@dpu.edu.krd
}

\begin{abstract}
Skin cancer is among the primary cancer types that manifest due to various dermatological disorders, which may be further classified into several types based on morphological features, color, structure, and texture. The mortality rate of patients who have skin cancer is contingent on preliminary and rapid detection and diagnosis of malignant skin cancer cells. Limitations in current dermoscopic images, including shadow, artifact, and noise, affect image quality, which may hamper detection effort. Attempts to overcome these challenges have been made by analyzing the images using deep learning neural networks to perform skin cancer detection. In this paper, the authors review the state-of-the-art in authoritative deep learning concepts pertinent to skin cancer detection and classification.
\end{abstract}

Keywords: skin lesion classification, skin cancer, melanoma detection, convolutional neural networks, Deep Learning.

Received: March $2^{\text {nd }}, 2021 /$ Accepted: March 29 $9^{\text {th }}, 2021 /$ Online: March 31 ${ }^{\text {st }}, 2021$

\section{INTRODUCTION}

Cancer is a concoction of several disease states whereby body cells perform out-of-control replication and division that may spread viciously and invade neighboring tissues. Cancer forms vary, in which skin cancer has the highest chance of occurrences with a significant malignancy risk [1]. As the most common cancer type, skin cancer is generally classified into melanoma and non-melanoma categories [2, 3]. Malignant lesion type involves a substantial cost to healthcare and contributes to cancer morbidity. Due to these factors, researchers have invested research efforts towards developing algorithms that possess high accuracy with flexibility in detecting early melanoma. Early detection is crucial as cancerous melanocyte cells could perform cell division uncontrollably, invade nearby tissues, and become highly metastasized, leading to high mortality rates [4]. Physicians often rely on Dermiscopy or Epiluminicence Microscopy (ELM) method to determine malignant or benign skin lesions.

Such a method utilizes a dermatoscopy, which amplifies medical pattern visualization through a lens with a light source to highlight colors, veils, pigmented networks, globs, ramifications, and various others. Dermatologists identify melanoma through assessing visible features such as Asymmetrical form, Border anomaly, Color discrepancy, Diameter, and Evolution (ABCDE rule) [5]. Despite this, caution is exercised due to limitations in dermoscopic images that need to be addressed. Since the emergence of image processing techniques, improvements have been pursued to improve Computer-Aided Detection (CAD) systems and approaches in Pigmented Skin Lesion (PSL) segmentation and classification, leading to easing patients' early diagnoses with less invasive or traumatizing medical procedures $[6,7]$. Stateof-the-art advances in machine learning, particularly deep neural networks, have made great strides in various areas. A required field in which deep neural networks may be exploited is processing medical images such as skin lesion images in the medical field. The skin lesion is referred to as anomalies in skin appearances such as visible signs of sore, abnormal lump, or colored skin color. Physicians may use machine learning techniques to recognize and classify skin lesions in images before making decisions that would affect patients' health $[8$, 9]. In general, there are four primary machine learning steps in the detection and diagnosis of melanoma cancer, comprising preprocessing of images, segmentation, feature extraction, and classification of images capturing the lesions (see Fig. 1) [10]. Litjens et al. 2017 [11] reviewed authoritative deep learning concepts relevant to analyzing medical images, which covered images' classification, detection of objects, segmentation, registration, and several others. In 2018 Okur et al. [12] carried out a survey focusing on melanoma and methods used to visualize and detect melanoma autonomously in dermoscopic images. In 2019, Kassani et $a l$. conducted a comparative study of the latest deep learning approaches on 
dermoscopic images in classifying skin lesions [4]. In another study in the same year, Munir et al. (2019) presented their chronological advancements in cancer diagnosis and the application of machine learning on medical images in [13]. In this paper, the authors aim to review the state-of-the-art deep learning architectures with dominant concepts and challenges of a cancer diagnosis on dermoscopic images.

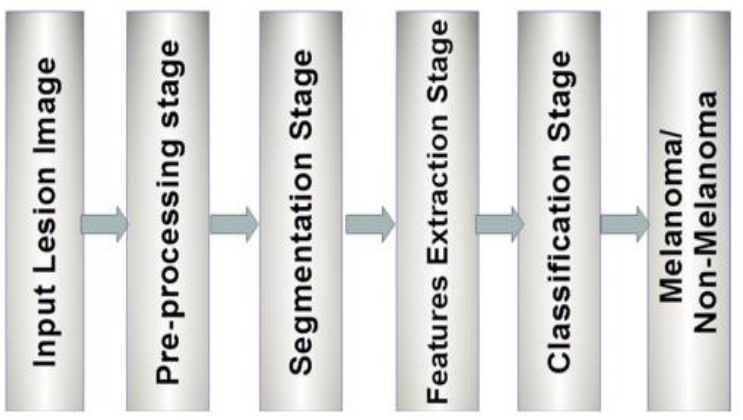

Fig. 1 Pipeline process of a melanoma cancer diagnosis from skin lesion image analysis [10]

The rest of the paper is organized as follows: Section 2 presents skin cancer types and summarizes dermoscopic image datasets. Section 3 illustrates a detailed description of the steps involved in the diagnosis of skin lesion images. Meanwhile, Section 4 lays authoritative Deep Convolutional Neural Networks (DCNN) techniques and performance evaluation measures. Finally, Section 5 offers a discussion and concludes this review.

\section{BACKGROUND THEORY}

\section{A. Skin Cancer Types}

The occurrence of skin cancer is associated with UV ray exposure from the sun, contributing to skin cells' DNA impairment. Gene mutations would trigger when DNA is damaged, whereby skin cells multiply excessively, leading to tumors' formation. In addition to UV ray exposure, genetic defects are also a contributing factor for skin cancer [14]. Fig. 2 illustrates the effect of skin cancer on the skin $[15,16]$. Lesions on the skin could be due to different causes such as allergies, cancerous cells, etc. However, among these, skin lesions due to cancerous cells are perilous. In the worst case, certain manifestations of cancerous skin lesions are fatal. Among cancer-related lesions, melanoma contributes to an $8 \%$ mortality rate, which is considered a significantly high rate.

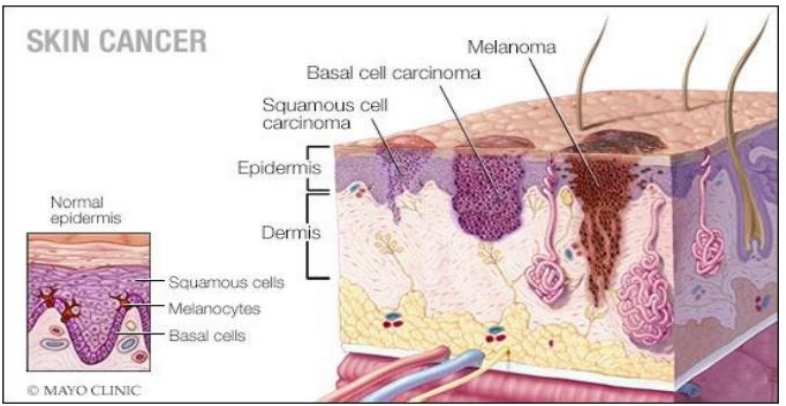

Fig. 2. Skin cancer affected skin image

Skin cancer is classified into two categories:

- Melanoma Skin Cancer (MSC)

- Non-Melanoma Skin Cancer (NMSC)

The following are eight categories of cancerous skin lesions shown in Fig. 3:

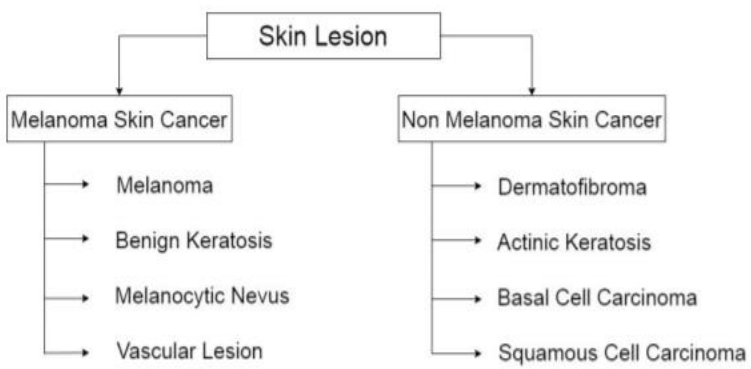

Fig. 3. Classification of skin lesions

$\mathrm{ABCDE}$ rule has been generally utilized to diagnose skin cancers, as following [17] clinically:

- A: Asymmetry property. Two halves of skin lesions are assessed for similarity in aspects of edges, shape, and color.

- B: Border property. Skin lesion edges are assessed in appearance to see if they are well-defined and smooth. Otherwise, the lesion is likely to be melanoma if the edges are jagged, fuzzy, and uneven.

- C: Color property. Melanoma visibly shows color contrast between different skin regions with shades varying from black, red, brown, and tan.

- D: Diameter property. Skin lesion diameter that exceeds $6 \mathrm{~mm}$ generally tells a sign of melanoma.

AS DESCRIBED, the ABCDE rule is typically carried out by conducting a physical assessment of skin with the naked eye. Despite this, there have been cases whereby melanoma appears identical to benign lesions while following the ABDCE rule (see Fig.4), leading to erroneous judgment. On the other hand, different clinical assessments such as biopsy are prone to errors. Specialists' accuracy in predicting whether a skin lesion is malignant or otherwise falls in the range of $49 \%$ to $81 \%$, where a third of melanomas have been inaccurately declared as benign lesions. These low accuracies have prompted the application of dermoscopy [17-19]. 


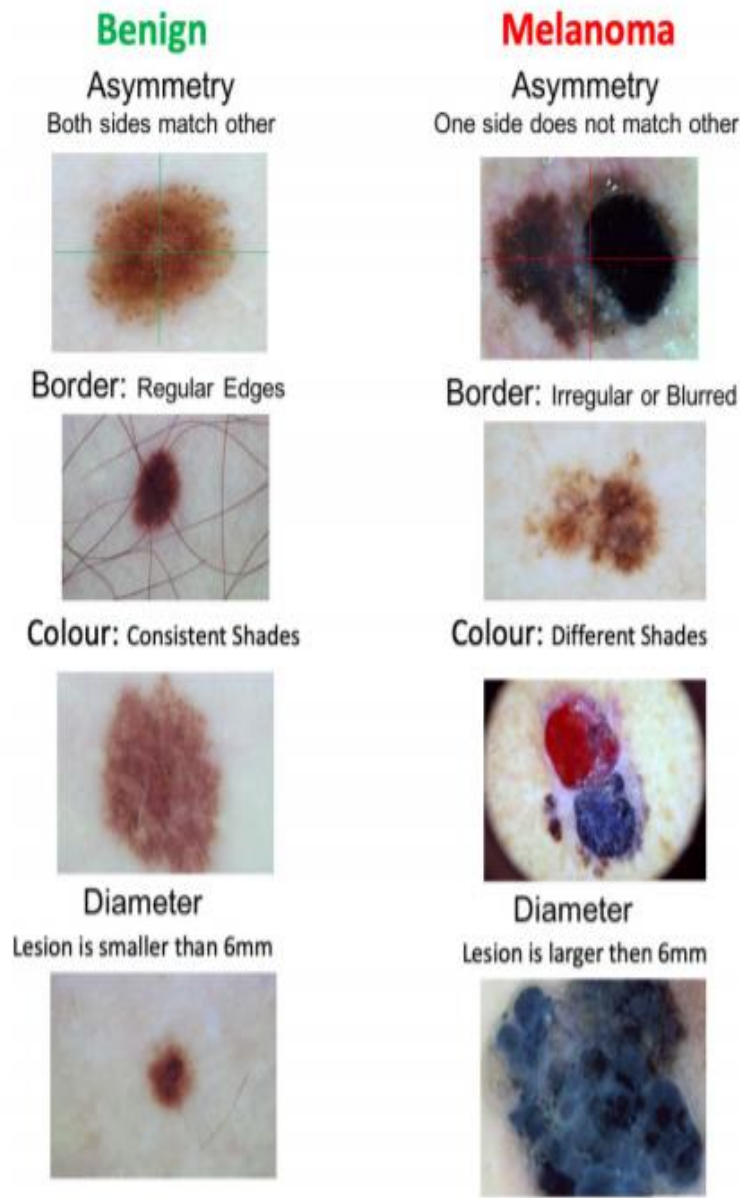

Fig.4. Lesion diagnosis by dermatologists. ABCD criteria for lesion diagnosis focuses on finding the certain properties of lesions[20].

\section{B. Dermoscopic Image Datasets}

Table I list`s both commercial and open-access, wellknown datasets containing dermoscopic images that have been utilized in the studies of segmentation and classification. Dermot and Atlas of Dermoscopy are commercial datasets, while the remaining as listed in Table I are accessible for free to be used for scholarly purposes. In terms of dataset size, commercial datasets are small in comparison to the biggest open-access dataset size. The largest dataset from the International Skin Imaging Collaboration ISIC repository contains 25,331 images. Within ISIC nests, several subdatasets comprising ISIC 2016, ISIC 2017, ISIC 201811, and ISIC 2019 datasets. The growth in dataset size in ISIC over the years has allowed researchers to conduct fully and semiautonomous methods in performing segmentation and classification. These openly-accessible free datasets comprising dermoscopic images were obtained from varying clinical institutions. Respective ground truth of the obtained datasets had either been verified by expert consensus, pathologists, or supplementary techniques [21]. Despite the laudable diversity of image samples, most openly available datasets still lack highly diversified skin lesion samples covering vast populations and extremely rare cases (out-ofdistribution category) [22-24].
TABLE I: WELL-KNOWN DATASETS FOR DERMOSCOPIC IMAGE CLASSIFICATION AND SEGMENTATION.

\begin{tabular}{|c|c|c|c|}
\hline Dataset & $\begin{array}{c}\text { Total } \\
\text { Images }\end{array}$ & $\begin{array}{c}\text { Number of } \\
\text { Classes }\end{array}$ & Reference \\
\hline Dermofit & 1300 & 10 & {$[25]$} \\
\hline Atlas of Dermoscopy & 1024 & 7 & {$[26]$} \\
\hline PH2 & 200 & 2 & {$[27]$} \\
\hline Derm7pt & 1011 & 5 & {$[28]$} \\
\hline ISBI-2017 & 2750 & 3 & {$[29]$} \\
\hline \multirow{2}{*}{ ISIC } & 900 & 2 & {$[30,31]$} \\
\cline { 2 - 4 } & 2000 & 7 & {$[21]$} \\
\cline { 2 - 4 } & 25331 & 9 & {$[31]$} \\
\hline
\end{tabular}

\section{Skin Lesion Images Diagnoses Steps}

\section{Preprocessing}

Detection procedures require preprocessing of raw data as the data may contain noise [32-34]. In skin lesion images, captured images typically carry noises encompassing uneven illumination, skin surface light reflection, and hair. Such noises may affect the performance of segmentation undesirably and thus, need to be resolved $[6,35]$. One of the techniques to resolve noises in preprocessing is the usage of filters. Filters such as adaptive wiener filter, Gaussian filter, adaptive median filter, mean filter, and median filter may be used to purge noises including salt and pepper noise, Poisson noise, Gaussian speckle noise. The presence of noise such as hair might lead to the inaccurate classification of skin lesions. Relevant preprocessing on images should be carried out to remove or adjust noises through strategies such as localization, normalization, hair removal, image smoothing, color correction, removal of the vignette, and adjustment of contrast. Greater accuracy would be obtained with an appropriate combination of preprocessing tasks [13, 36-38].

\section{Segmentation}

Detection of melanoma through automation is initiated with the segmentation of the lesion. This phase is deemed the least difficult to be understood but is also a critical phase. This is because skin lesions' segmentation has a knock-on effect on the segmentation of clinical features and generation of features for classification[39]. The phase involves separating the background from the lesion (i.e., skin) and other artifacts. The separation is often manifested in the form of a binary image (also known as a binary mask), whereby labels are assigned to a region of background skin removed and the lesion region that will be analyzed further. Upon separation of lesion region from the background, clinical features would undergo segmentation. The segmentation would reveal distinct global features, including border irregularity and asymmetry information. Fig.6 illustrates the segmentation of lesions with much success. Segmentation is considered successful if the background is removed entirely.

On the other hand, a less effective segmentation would sometimes retain pixels of background within the region where the lesion is segmented, particularly in the vicinity of borders. This could have an adverse effect as inaccurate local 
and global border features would be produced, in addition to extraction of color features that are purposeless in the subsequent feature segmentation phase. Numerous techniques to achieve lesion segmentation, essentially a task of image segmentation, have been proposed by researchers $[12,40]$.
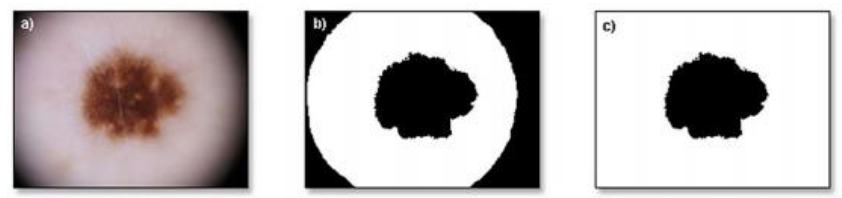

Fig. 5. Color space optimization system with clustering-based histogram thresholding for lesion segmentation: (left) block diagram of optimization procedure, and (right) color channels used in color space transform [12].

\section{Features extraction}

Extraction of features holds the key to an effective classification system[41, 42]. In this phase, crucial information in the skin lesion image is extracted, which then will be used to describe a lesion, leading to a successful classification of whether the lesion is a melanoma or otherwise [43]. In recent years, substantial advancement has been achieved in the computer vision field utilizing larger datasets. Researchers that use deep Learning have extracted a greater extent of features in various layers $[44,45]$.

\section{Classification}

Medical image analysis has shown a greater inclination towards applying machine learning methods, particularly deep learning-based algorithms attributed to favorable outcomes. For instance, a deep convolution algorithm can exhibit greater accuracy, become more objective-oriented, and generate reproducible results once training is conducted successfully. Therefore, this review offers a glance at state-of-the-art on skin cancer classification [46, 47].

\section{DeEP LEARNING-BASED TECHNIQUeS}

Deep learning-based classifiers' application is widespread as they can exceed human capability in performing classifying tasks for objects in general [48, 49]. For instance, Deep Neural Networks can compute complex tasks attributed to nonlinear neuron processing and greater prediction power, rendering them suitable to be applied in clinical images[50, 51]. Most recent advancements in deep learning models include VGG, AlexNet, ResNet, and Xception [38]. Various research works have also utilized these models in Computer-Aided Diagnose $(\mathrm{CAD})$ as their performance is efficient.

\section{A. Deep Convolutional Neural Networks (DCNNs)}

DCNNs took inspiration from the biological visual cortex to perform analysis on visual imagery based on computer models. The models have shown great reliability, accuracy, and efficiency in classifying images. In various complex classification tasks involving natural and medical visual imageries for classifying diseases, the models had successfully attained close to baseline humans' results[ $[11,52]$. The CNN, commonly referred to as ConvNet, is a distinct category of feedforward neural networks, where the outputs do not form feedback and hence will not be fed to itself. The CNN comprises a convolutional layers stack, accompanied by a pooling layer responsible for extracting input data's features. Subsequently, high-level feature maps are produced upon each convolution level. As the reduction of feature maps' parameters is critical for efficiency, pooling layers summarize their information. Fig. 6 represents an illustration of a conventional convolutional neural network [53]. A CNN with five layers has been proposed in [54] to classify skin lesions into three classes, including melanoma class that is a form of terminal skin cancer. With training and testing on dermoscopic images from the $\mathrm{PH} 2$ dataset, the proposed $\mathrm{CNN}$ classifier attained an accuracy rate of $95 \%$.

Moreover, a greater accuracy rate of $97.78 \%$ was obtained in[55] when the number of layers in CNN was increased to 14 layers on dermoscopic images from the ISIC dataset. Despite this, greater resources and computation complexity would be expended when layers are increased. CNN has also been used to classify rashes and skin cancer detection[15]. [56] utilized Deep Learning Studio to exploit a Model-Driven Architecture to construct Deep Learning. The researchers introduced the studio suite features, which were used to facilitate the development of a Deep Learning Model. The researchers further elaborated dermal cell image preparation and demonstrated the DLS model in detecting cancer cells. The model obtained an AUC of $99.77 \%$ to detect cancer cells based on their medical images. In [57], a Deep Residual Network (DRN) was applied in the classification phase's training model. A fair accuracy was attained at the classification phase when Fully Convolutional Network (FCRN) was utilized. However, given that the application is meant for medical image classification, the rate of false positives must be reduced to be effective for medical applications. Towards achieving this, emphasis on the recall factor must exceed the emphasis on overall accuracy in conducting studies.

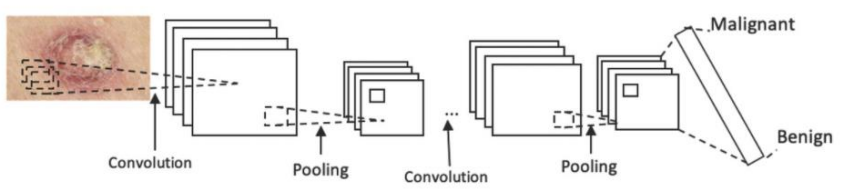

Fig. 6. The architecture of a typical convolution neural network

\section{B. Deep Learning and Classical Machine Learning Techniques (Hybrid Approach)}

Several studies have proposed approaches that would facilitate skin lesion categories by inspecting established features or exploiting well-known deep learning models' capability. Despite this progress, a combination of established features with deep learning models in an integrated framework has been sparsely reported in the literature. Constructing an effective strategy in extracting local and global features would greatly improve differentiating between normal skin and certain lesions. Motivated by these factors, researchers in [58] introduced an integrated model that extracts global-local 
features through combining local binary pattern(LBP) features and deep Conv-features. Various studies have also attempted the combination of Local-DNN and Global-DNN in the hunt for superior outcomes. Researchers typically leverage segmentation models to purge background noise in the detection of apparent features from visual imagery. Researchers in [59] implemented a hybrid approach using three models for predicting lesions. The approach involves a CNN model and two conventional machine learning classification models trained using a collection of features that describe skin lesion's color, texture, and borders. The models were then hybridized with the objective of performance improvement through votes of the majority.

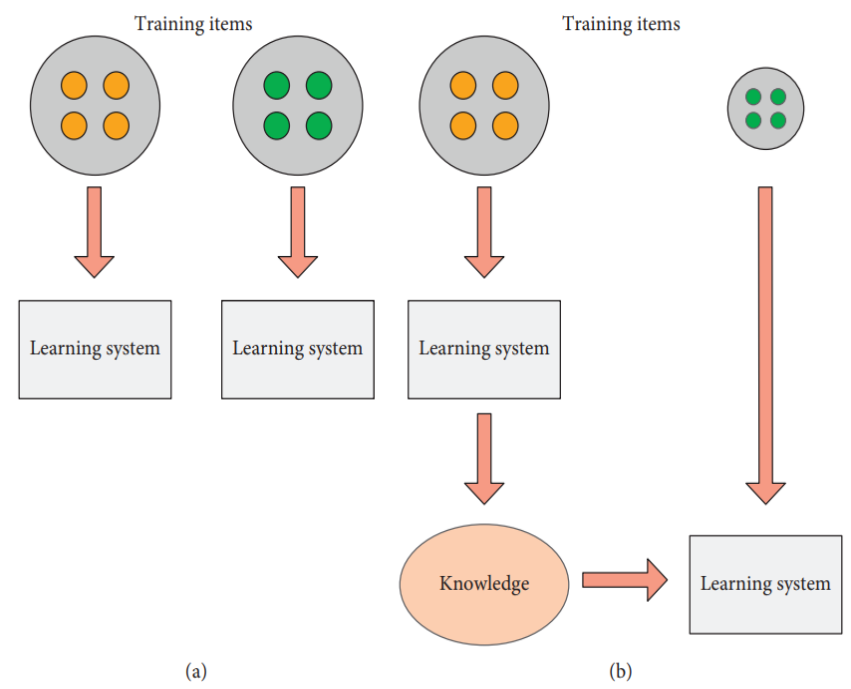

Fig. 7. (a) Traditional machine learning vs. (b) transfer learning

\section{Deep Learning with Transfer Learning and Image Augmentation}

Transfer learning involves applying a sub-machine learning technique that learns new tasks based on previous knowledge of learned tasks. Adaptation of domain and transfer learning generally refers to improving generalization capability in a subsequent setting based on a previous single set training [60]. Significant dataset size is required to train a new DCNN model, which poses an obstacle to the model as existing skin lesion datasets lack labeled images in large datasets. Theoretical understanding of transfer learning is essential in solving the obstacle. Fig. 7 illustrates key differences between conventional machine learning and transfer learning. Transfer learning can address small datasets, leading to augmenting model learning performance [61, 62]. Fine-tuning of trained models allows adaptation to problems to be achieved [63]. Besides, strategies to augment small datasets have been adopted broadly in classifying raw images and melanoma. Augmentation of small datasets solves the issue of data scarcity prone to overfitting in the classification of melanoma. The fundamental concept in the augmentation of images lies in the convention that data annotation does not alter labels' semantic meaning [64]. Fig. 9 illustrates augmented images created through the application of several data augmentation approaches.

Researchers in [65] proposed automation of discriminative features extraction via ROIs. The system is also capable of addressing an imbalance of classes through augmenting data. As the proposed system utilized transfer learning, improvement of low-level features learning of the AlexNet model was achieved with an efficient false positives reduction in the CAD system. Challenges in classifying multiple classes may be resolved into a two-class classification type via transformation using the Error-Correcting Output Codes (ECOC) method. Work in [66] applied a pre-trained AlexNet CNN model. A classifier based on ECOC SVM was implemented in classifying four classes of skin cancer (i.e., Melano, Squamous cell carcinoma, Basal cell carcinoma, and Actinic Keratoses). In[67], ImageNet's pre-trained data, including DENSENET121, RESNET50, and VGG11, were used in a transfer learning model.

Consequently, this has led to improved accuracy at a $90 \%$ rate in training with a modest rate of losses. Meanwhile, transfer learning and DNN were applied in [68]along with data augmentation and fine-tuning. AlexeNet was utilized with transfer learning through substituting the final layer with a softmax in the classification of three lesion classes (i.e., atypical nevus, common nevus, and melanoma). PH2 dataset was utilized for training and testing with an accuracy rate achieved of $98.61 \%$. Researchers tested CNN variants in [69], including DenseNet, EfficientNet, MobileNet, and Inception V3. The findings indicated that CNN Xception obtained a superior accuracy rate at $89 \%$. A DCNN architecture for binary classification supporting multiple classes was proposed in[70] that offers greater outcome reliability in significant probabilities. An identical CNN architecture (GoofgLeNet Inception-v3) trained classification of multiple and binary classes concurrently. As reported in [71], Inception-v3 achieved superior performance in comparison to ResNet-101 architecture. DenseNet121, ResNet50, and VGG11 models are used in [59] by employing ImageNet's pre-trained data. The models augmented dataset size, which led to an improved model efficiency. In training, an accuracy rate of $90 \%$ was obtained with a modest loss rate. In [72], PH2 and HAM10000 datasets were employed in pre-trained innovative models (i.e., VGG16 and Mobilenet), subjected to two conditions (i.e., without augmentation and with augmentation). A tailor-made deep learning architecture was then constructed and evaluated against the two innovative models' performance. A conjecture that a thoughtful model design from the ground up would perform just as fine. From the results, Mobilenet and the tailormade model fared well inaccuracy rate performance. Despite this, the result indicated an insignificant effect of data augmentation in comparison to non-augmented data in classification. 


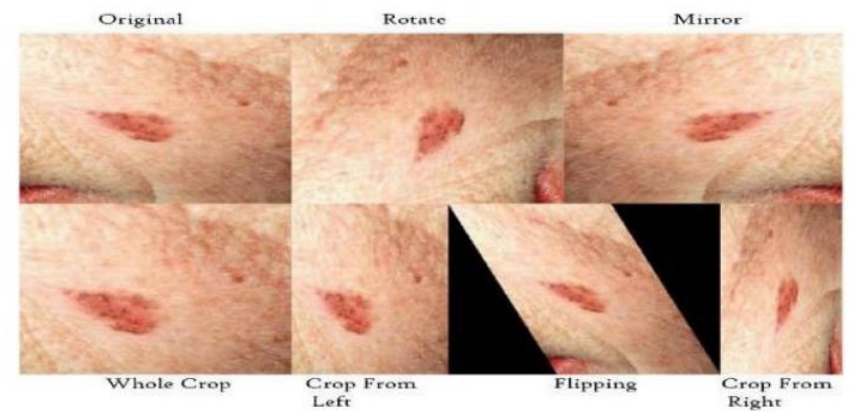

Fig. 8. Sample of melanoma augmented images [57].

Predominantly, past classification solutions inclined towards employing complex and sophisticated models to improve accuracy rate in detection. Research evidence on intraclass dissimilarity and inter-class similarity in lesion features is rather sparse in the literature. Employing a sophisticated model with a large computation overhead might render challenges to applicability in the real world. Motivated by using a less sophisticated model, researchers in [73]. Put forth a discriminant dermoscopy image lesion recognition model. The model extracts discriminant features by employing a pre-trained lightweight network, which builds a network of dermoscopy image lesion classification branches and a network of lesion feature discriminant branches. The model executes a shared training for the individual branch network, consequently enabling lesion type to be classified while lesion feature similarity is also established concurrently. As a result, this allows a greater extraction capability for discriminative lesion features. Ensemble-based approaches [74-76] are commonplace among researchers to enhance individual approaches' accuracy rate performance.

\section{Deep Learning and Generative Adversarial Network (GAN)}

Goodfellow et al. [77] pioneered Generative Adversarial Networks (GAN) into the deep learning field. As the name implies, GAN is a generative model that carries out training through an adversarial deep neural network setting. In operation, the GAN performs a generative model learning for data distribution by employing an adversarial strategy. Since its inception, the GAN has been recognized as the most dominant generative model explored in artificial intelligence research[78]. Despite that, such deep architecture demands actual samples to be used for training to learn meaningful representations. As mentioned previously, large datasets for medical imaging are currently unavailable to facilitate supervised Learning. This is largely due to the arduous process that consumes time and prohibitive cost involved in the labeling and acquiring datasets that often require sophisticated tools and expert human judgment. Consequently, the absence of readily accessible largescale datasets has prevented researchers from fully leveraging deep learning possibilities in medical imaging applications [79]. The work in[80] utilized GANs to generate imagery with close resemblances to dermoscopic images. The generated images are subsequently included in augmented trained data towards enhancing deep CNN's performance in classifying skin lesions. Discriminator and generator as outlined in algorithm one may be attained by using formal expressions [69], expressed in Equation (1) and (2), respectively, as follows:

$$
\begin{aligned}
\nabla \theta_{d} & =\frac{1}{m} \sum_{n=1}^{m}\left[\log D\left(x^{i}\right)+\log \left(D\left(G\left(z^{i}\right)\right)\right)\right] \\
\nabla \theta_{g} & =\frac{1}{m} \sum_{n=1}^{m}-\log \left(D\left(G\left(z^{i}\right)\right)\right)
\end{aligned}
$$

Where $\mathrm{z}$ is noise exhibiting uniform or normal distribution, $\mathrm{G}$ is the generator employed in image creation contingent on $x=G(z)$. While $m$ is the count of noise and samples realized as a result of data generation, $\mathrm{D}(\mathrm{x})$ is a function responsible for computing probability that $\mathrm{x}$ descended from data instead of generator distribution. Besides, $\nabla$ it is stochastic gradient descent employed in training GANs adhering to $\theta \mathrm{d}$ and $\theta \mathrm{g}$ parameters. About algorithm one and compensating the training data scarcity, Sedigh et al.[81] put forth a CNN algorithm, a GAN variant towards generating mock images of skin cancer. Fig. 9 presents the GAN algorithm structure. Work in[82] proposed GAN to generate style-based mock skin lesion images with a close reference to the earliest proposed GAN algorithm. The proposed work alters style control structure and noise input in the generator. It modifies generator and discriminator intending to generate fine-quality mock images of skin lesions with efficiency in mind. Meanwhile, in classifying images, a classifier's construction on the pre-trained deep neural network expanded the transfer learning theory. Mock skin lesion images produced from the proposed work subsequently become additions to the existing training set, contributing to superior performance classification tasks.

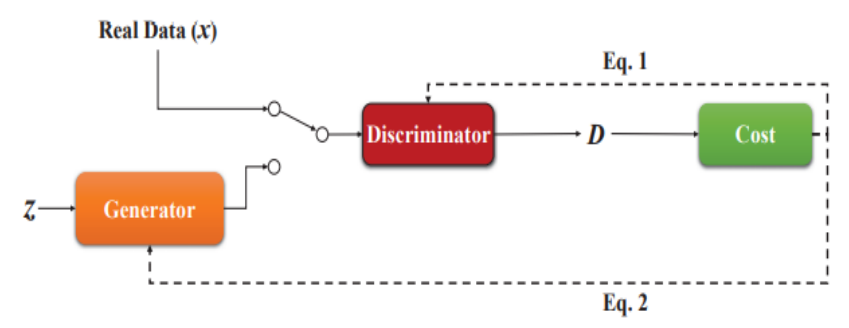

Fig. 9. The GAN algorithm structure[81].

\section{E. Performance Evacuation}

In predicting skin lesions employing DCNN architectures, several measures may evaluate performance, including accuracy, sensitivity, and specificity. Referring to Equation (3)-(6) [83-85], true positives (TP) are positive instances that are predicted correctly, false negatives $(F N)$ are negative instances that are predicted incorrectly. Meanwhile, true negatives $(T N)$ are negative instances that are predicted correctly. Finally, false positives $(F P)$ are positive instances that are predicted incorrectly. Recall or sensitivity refers to correctly classified skin lesions [86]. Classification applications in the medical field require great sensitivity as it represents the measure of the system's worthiness. Sensitivity 
is formally expressed as Equation (3): Sensitivity or True Positive Rate

$$
\mathrm{TPR}=\frac{\mathrm{TP}}{(\mathrm{TP}+\mathrm{FN})}=
$$

Specificity refers to the measure of non-skin lesion labels that have been classified successfully, which is formally expressed as Equation (4): Specificity or True Negative Rate

$$
\mathrm{TNR}=\frac{\mathrm{TN}}{(\mathrm{TN}+\mathrm{FP})}
$$

Precision or positive predictive value quantifies the percentage of correctly classified labels that are truthfully positive, which is formally expressed as Equation (5): Positive Predictive Value

$$
\mathrm{PPV}=\frac{\mathrm{TP}}{(\mathrm{TP}+\mathrm{FP})}
$$

Accuracy (ACC) quantifies the count of correctly classified skin lesions divided by the total number of skin lesions, which is formally expressed as Equation (6):

$$
\text { Accuracy } \%=\frac{\mathrm{TP}+\mathrm{TN}}{(\mathrm{TP}+\mathrm{TN}+\mathrm{FP}+\mathrm{FN})}
$$

\section{DISCUSSION}

Skin cancer continues to impact communities worldwide as a deadly disease. Early detection is important to increase the patients' survival chance as the disease is fatal. In recent years, research on deep learning models in detecting skin cancer has grown substantially, given that the models offer the concept of error-less decision-making for medical applications. Most recently, research effort has slowly progressed towards deep convolutional neural network architectures. From what has been reviewed above, it is clear that considering transfer learning (pertained network), finetuning, ensemble method, data generation, and augmentation aim to mitigate insufficiency of labeled data prone to overfitting and generally improving the performance of skin lesion classification in CAD systems. Table II summarizes the

\begin{tabular}{|c|c|c|c|c|c|c|}
\hline Ref. & Year & Author(s) & Objective & Model & Dataset & Accuracy \\
\hline$[15]$ & 2020 & Subha, S., et al. & $\begin{array}{l}\text { Detecting and distinguishing between skin } \\
\text { cancer from rashes images by employing CNN. }\end{array}$ & $\mathrm{CNN}$ & $\mathrm{A} / \mathrm{N}$ & $80.2 \%$ \\
\hline [54] & 2020 & Alkarakatly, T., et al. & $\begin{array}{l}\text { Diagnosing three skin lesion classes, including } \\
\text { malignant skin cancer lesion, melanoma. }\end{array}$ & 5-layer CNN & $\mathrm{PH}^{2}$ & $95 \%$ \\
\hline$[55]$ & 2019 & Mohamed, A., et al. & $\begin{array}{l}\text { Autonomous dermoscopic detection pattern that } \\
\text { employs deep convolutional neural networks. }\end{array}$ & 14-layer CNN & (ISIC) & $97.78 \%$ \\
\hline$[56]$ & 2020 & $\begin{array}{l}\text { Kadampur, M.A. et } \\
\text { al. }\end{array}$ & $\begin{array}{l}\text { Construct deep learning models for dermal cell } \\
\text { image classification and skin cancer detection } \\
\text { without prior knowledge of employing Deep } \\
\text { Learning Studio }\end{array}$ & DLS & HAM10000 & AUC of $99.77 \%$ \\
\hline$[57]$ & 2020 & Vinay, B., et al. & $\begin{array}{l}\text { Diagnosing melanoma and non-melanoma } \\
\text { images in a two-stage network. }\end{array}$ & $\begin{array}{l}\text { Deep Residual } \\
\text { Network (DRN) }\end{array}$ & ISIC & $88.7 \%$. \\
\hline [59] & 2020 & Daghrir, J., et al. & $\begin{array}{l}\text { Detecting melanoma skin cancer through a } \\
\text { hybrid method that leverages the superiority of a } \\
\text { particular method. }\end{array}$ & $\begin{array}{l}\text { CNN, KNN, and } \\
\text { SVM }\end{array}$ & ISIC & $88.4 \%$ \\
\hline \multirow[t]{2}{*}[65]{} & \multirow[t]{2}{*}{2020} & \multirow[t]{2}{*}{ Ashraf, R., et al. } & \multirow{2}{*}{$\begin{array}{l}\text { Transferring initial low-level feature layers of } \\
\text { AlexNet model and evaluating ROI with } \\
\text { augmentation for attaining ideal results. }\end{array}$} & \multirow[b]{2}{*}{ AlexNet } & DermIS & $97.9 \%$ \\
\hline & & & & & DermQuest & $97.4 \%$ \\
\hline$[66]$ & 2018 & Dorj, U.-O., et al. & Diagnosing four skin cancer classes. & Alex Net & $\begin{array}{l}\text { collected } \\
\text { from the } \\
\text { internet }\end{array}$ & $94.2 \%$ \\
\hline
\end{tabular}
reviewed recent researches on models of skin cancer detection and classification.

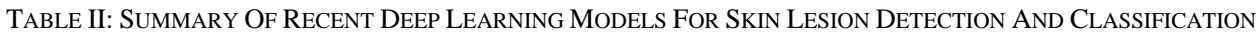


Saeed \& Zeebaree. / Journal of Applied Science and Technology Trends Vol. 02, No. 01, pp. 41 -51 (2021)

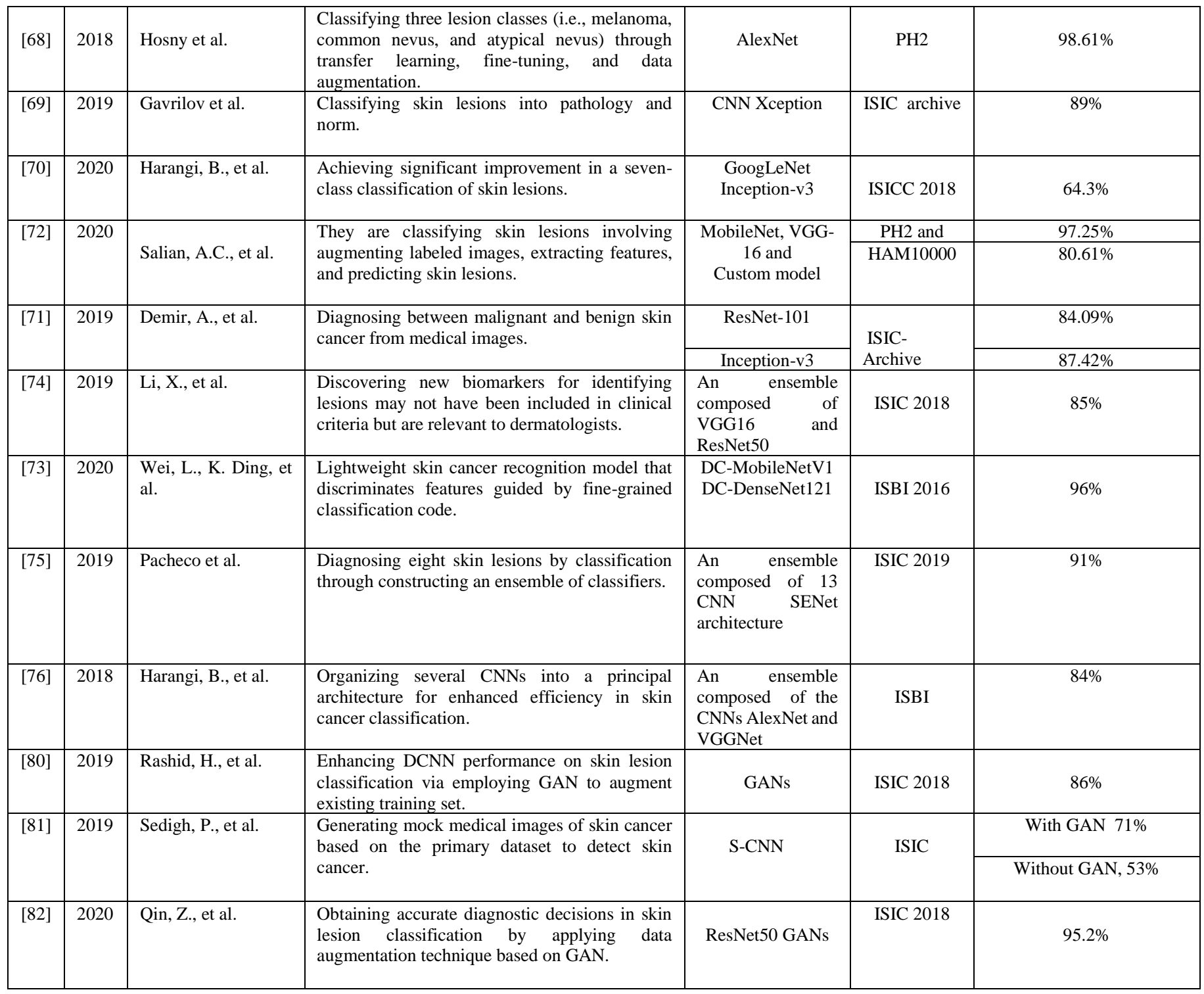

\section{CONCLUSION}

Skin cancer remains deadly cancer affecting populations worldwide. Since the disease is fatal, early detection is critical towards improving the survival chance of patients. Admittingly, a shortage of qualified experts and sophisticated medical equipment have affected the fight against skin cancer. This paper looked into the latest research efforts to advance skin cancer detection and classification through variants of DCNN architectures. Transfer learning, fine-tuning, ensemble approach, data generation, and augmentation are all effectively used for minimizing insufficiency of labeled data, which is prone to overfitting. It also contributes to improving the efficiency of skin lesion classification in CAD systems in general. Application areas such as medical imaging whereby largescale training datasets are technically unavailable, building a rich dataset covering skin lesion samples from vast populations that includes extremely rare cases (out-ofdistribution detection) is crucial to tackling the problem of scarcity of labeled data, which will also facilitate the advancement of skin cancer detection field further.

\section{REFERENCES}

[1] M. A. Albahar, "Skin lesion classification using convolutional neural network with novel regularizer," IEEE Access, vol. 7, pp. 38306-38313, 2019.

[2] H. Xu, C. Lu, R. Berendt, N. Jha, and M. Mandal, "Automated analysis and classification of melanocytic tumor on skin whole slide images," Computerized medical imaging and graphics, vol. 66, pp. 124-134, 2018.

[3] A. Namozov and Y. Im Cho, "Convolutional neural network algorithm with parameterized activation function for melanoma classification," in 2018 International Conference on Information and Communication Technology Convergence (ICTC), 2018: IEEE, pp. 417-419.

[4] S. H. Kassani and P. H. Kassani, "A comparative study of deep learning architectures on melanoma detection," Tissue and Cell, vol. 58, pp. 7683, 2019.

[5] I. A. OZKAN and M. KOKLU, "Skin Lesion Classification using Machine Learning Algorithms," International Journal of Intelligent 
Systems and Applications in Engineering, vol. 5, no. 4, pp. 285-289, 2017.

[6] J.-A. Almaraz-Damian, V. Ponomaryov, S. Sadovnychiy, and H. Castillejos-Fernandez, "Melanoma and nevus skin lesion classification using handcraft and deep learning feature fusion via mutual information measures," Entropy, vol. 22, no. 4, p. 484, 2020.

[7] K. M. Hosny, M. A. Kassem, and M. M. Foaud, "Classification of skin lesions using transfer learning and augmentation with Alex-net," PloS one, vol. 14, no. 5, p. e0217293, 2019.

[8] H. A. Hassan, M. B. Jan, Z. Ahmad, F. Tahira, and M. A. Khan, "Skin Lesion Classification Using Deep Learning Techniques."

[9] M. N. Bajwa et al., "Computer-Aided Diagnosis of Skin Diseases Using Deep Neural Networks," Applied Sciences, vol. 10, no. 7, p. 2488, 2020.

[10] A. Adegun and S. Viriri, "Deep learning techniques for skin lesion analysis and melanoma cancer detection: a survey of state-of-the-art," Artificial Intelligence Review, pp. 1-31, 2020.

[11] G. Litjens et al., "A survey on deep learning in medical image analysis," Medical image analysis, vol. 42, pp. 60-88, 2017.

[12] E. Okur and M. Turkan, "A survey on automated melanoma detection," Engineering Applications of Artificial Intelligence, vol. 73, pp. 50-67, 2018.

[13] K. Munir, H. Elahi, A. Ayub, F. Frezza, and A. Rizzi, "Cancer diagnosis using deep learning: a bibliographic review," Cancers, vol. 11, no. 9, p. 1235, 2019.

[14] S. Pathan, K. G. Prabhu, and P. Siddalingaswamy, "Techniques and algorithms for computer aided diagnosis of pigmented skin lesions-A review," Biomedical Signal Processing and Control, vol. 39, pp. 237262, 2018.

[15] S. Subha, D. J. W. Wise, S. Srinivasan, M. Preetham, and B. Soundarlingam, "Detection and Differentiation of Skin Cancer from Rashes," in 2020 International Conference on Electronics and Sustainable Communication Systems (ICESC), 2020: IEEE, pp. 389393.

[16] T. Satheesha, D. Satyanarayana, M. G. Prasad, and K. D. Dhruve, "Melanoma is skin deep: a 3D reconstruction technique for computerized dermoscopic skin lesion classification," IEEE journal of translational engineering in health and medicine, vol. 5, pp. 1-17, 2017.

[17] H. Nadipineni, "Method to Classify Skin Lesions using Dermoscopic images," arXiv preprint arXiv:2008.09418, 2020.

[18] R. B. Oliveira, A. S. Pereira, and J. M. R. Tavares, "Computational diagnosis of skin lesions from dermoscopic images using combined features," Neural Computing and Applications, vol. 31, no. 10, pp. 6091-6111, 2019.

[19] W. M. Abduallah and S. R. M. Zeebaree, "New Data hiding method based on DNA and Vigenere Autokey," Academic Journal of Nawroz University, vol. 6, no. 3, pp. 83-88, 2017.

[20] M. Goyal, A. Oakley, P. Bansal, D. Dancey, and M. H. Yap, "Skin lesion segmentation in dermoscopic images with ensemble deep learning methods," IEEE Access, vol. 8, pp. 4171-4181, 2019.

[21] P. Tschandl, C. Rosendahl, and H. Kittler, "The HAM10000 dataset, a large collection of multi-source dermatoscopic images of common pigmented skin lesions," Scientific data, vol. 5, p. 180161, 2018.

[22] M. E. Celebi, N. Codella, and A. Halpern, "Dermoscopy image analysis: overview and future directions," IEEE journal of biomedical and health informatics, vol. 23, no. 2, pp. 474-478, 2019.

[23] M. S. A. Mahbod, "Towards Improvement of Automated Segmentation and Classification of Tissues and Nuclei in Microscopic Images Using Deep Learning Approaches."

[24] N. A. Zebari, D. A. Zebari, D. Q. Zeebaree, and J. N. Saeed, "Significant features for steganography techniques using deoxyribonucleic acid: A review," Indonesian Journal of Electrical Engineering and Computer Science, vol. 21, no. 1, pp. 338-347, 2021.

[25] "DERMOFIT IMAGE LIBRARY." https://licensing.edinburghinnovations.ed.ac.uk/i/software/dermofit-imagelibrary.html?item=dermofit-image-library (accessed 4/1/2021.
[26] G. Argenziano, H. P. Soyer, V. De Giorgi, D. Piccolo, P. Carli, and M. Delfino, "Interactive atlas of dermoscopy (Book and CD-ROM)," 2000.

[27] T. Mendonça, P. M. Ferreira, J. S. Marques, A. R. Marcal, and J. Rozeira, "PH 2-A dermoscopic image database for research and benchmarking," in 2013 35th annual international conference of the IEEE engineering in medicine and biology society (EMBC), 2013: IEEE, pp. 5437-5440.

[28] J. Kawahara, S. Daneshvar, G. Argenziano, and G. Hamarneh, "Sevenpoint checklist and skin lesion classification using multitask multimodal neural nets," IEEE journal of biomedical and health informatics, vol. 23, no. 2, pp. 538-546, 2018.

[29] T. Akram et al., "A multilevel features selection framework for skin lesion classification," Human-centric Computing and Information Sciences, vol. 10, pp. 1-26, 2020.

[30] D. Gutman et al., "Skin lesion analysis toward melanoma detection: A challenge at the international symposium on biomedical imaging (ISBI) 2016, hosted by the international skin imaging collaboration (ISIC)," arXiv preprint arXiv:1605.01397, 2016.

[31] N. C. Codella et al., "Skin lesion analysis toward melanoma detection: A challenge at the 2017 international symposium on biomedical imaging (isbi), hosted by the international skin imaging collaboration (isic)," in 2018 IEEE 15th International Symposium on Biomedical Imaging (ISBI 2018), 2018: IEEE, pp. 168-172.

[32] S. Singhal and M. Jena, "A study on WEKA tool for data preprocessing, classification and clustering," International Journal of Innovative technology and exploring engineering (IJItee), vol. 2, no. 6, pp. 250-253, 2013.

[33] H. Dino et al., "Facial Expression Recognition based on Hybrid Feature Extraction Techniques with Different Classifiers," TEST Engineering \& Management, vol. 83, pp. 22319-22329, 2020.

[34] M. R. Mahmood, M. B. Abdulrazzaq, S. Zeebaree, A. K. Ibrahim, R. R. Zebari, and H. I. Dino, "Classification techniques' performance evaluation for facial expression recognition," Indonesian Journal of Electrical Engineering and Computer Science, vol. 21, no. 2, pp. 1761184, 2021.

[35] M. H. Jafari et al., "Skin lesion segmentation in clinical images using deep learning," in 2016 23rd International conference on pattern recognition (ICPR), 2016: IEEE, pp. 337-342.

[36] B. S. Osanaiye et al., "Network Data Analyser and Support Vector Machine for Network Intrusion Detection of Attack Type."

[37] O. F. Mohammad, M. S. M. Rahim, S. R. M. Zeebaree, and F. Y. Ahmed, "A survey and analysis of the image encryption methods," International Journal of Applied Engineering Research, vol. 12, no. 23, pp. 13265-13280, 2017.

[38] A. M. A. Brifcani and J. N. Al-Bamerny, "Image compression analysis using multistage vector quantization based on discrete wavelet transform," in 2010 International Conference on Methods and Models in Computer Science (ICM2CS-2010), 2010: IEEE, pp. 46-53.

[39] D. A. Zebari, H. Haron, S. R. Zeebaree, and D. Q. Zeebaree, "Enhance the Mammogram Images for Both Segmentation and Feature Extraction Using Wavelet Transform," in 2019 International Conference on Advanced Science and Engineering (ICOASE), 2019: IEEE, pp. 100-105.

[40] D. Q. Zeebaree, H. Haron, A. M. Abdulazeez, and S. R. Zeebaree, "Combination of K-means clustering with Genetic Algorithm: A review," International Journal of Applied Engineering Research, vol. 12, no. 24, pp. 14238-14245, 2017.

[41] H. I. Dino and M. B. Abdulrazzaq, "Facial expression classification based on SVM, KNN and MLP classifiers," in 2019 International Conference on Advanced Science and Engineering (ICOASE), 2019: IEEE, pp. 70-75.

[42] M. B. Abdulrazaq, M. R. Mahmood, S. R. Zeebaree, M. H Abdulwahab, R. R. Zebari, and A. B. Sallow, "An Analytical Appraisal for Supervised Classifiers' Performance on Facial Expression Recognition Based on Relief-F Feature Selection," in Journal of Physics: Conference Series, 2021, vol. 1804, no. 1: IOP Publishing, p. 012055. 
[43] Y. Filali, S. Abdelouahed, and A. Aarab, "An Improved Segmentation Approach for Skin Lesion Classification," Statistics, Optimization \& Information Computing, vol. 7, no. 2, pp. 456-467, 2019.

[44] M. A. Khan, M. Y. Javed, M. Sharif, T. Saba, and A. Rehman, "Multimodel deep neural network based features extraction and optimal selection approach for skin lesion classification," in 2019 international conference on computer and information sciences (ICCIS), 2019: IEEE, pp. 1-7.

[45] R. Zebari, A. Abdulazeez, D. Zeebaree, D. Zebari, and J. Saeed, "A Comprehensive Review of Dimensionality Reduction Techniques for Feature Selection and Feature Extraction," Journal of Applied Science and Technology Trends, vol. 1, no. 2, pp. 56-70, 2020.

[46] X. Du-Harpur, F. Watt, N. Luscombe, and M. Lynch, "What is AI? Applications of artificial intelligence to dermatology," British Journal of Dermatology, 2020.

[47] J. N. Saeed, A Survey Of Ultrasonography Breast Cancer Image Segmentation Techniques. Infinite Study, 2020.

[48] Y. Fujisawa, S. Inoue, and Y. Nakamura, "The possibility of deep learning-based, computer-aided skin tumor classifiers," Frontiers in Medicine, vol. 6, p. 191, 2019.

[49] K. B. Obaid, S. Zeebaree, and O. M. Ahmed, "Deep Learning Models Based on Image Classification: A Review," International Journal of Science and Business, vol. 4, no. 11, pp. 75-81, 2020.

[50] S. Albawi, Y. A. Abbas, and Y. Almadany, "Robust skin diseases detection and classification using deep neural networks," 2018.

[51] S. Albawi, T. A. Mohammed, and S. Al-Zawi, "Understanding of a convolutional neural network," in 2017 International Conference on Engineering and Technology (ICET), 2017: Ieee, pp. 1-6.

[52] S. R. Zeebaree, O. Ahmed, and K. Obid, "CSAERNet: An Efficient Deep Learning Architecture for Image Classification," in 2020 3rd International Conference on Engineering Technology and its Applications (IICETA), 2020: IEEE, pp. 122-127.

[53] R. Garg, S. Maheshwari, and A. Shukla, "Decision Support System for Detection and Classification of Skin Cancer using CNN," arXiv preprint arXiv:1912.03798, 2019.

[54] T. Alkarakatly, S. Eidhah, M. Al-Sarawani, A. Al-Sobhi, and M. Bilal, "Skin Lesions Identification Using Deep Convolutional Neural Network," in 2019 International Conference on Advances in the Emerging Computing Technologies (AECT), 2020: IEEE, pp. 1-5.

[55] A. Mohamed, W. A. Mohamed, and A. H. Zekry, "Deep learning can improve early skin cancer detection," International Journal of Electronics and Telecommunications, vol. 65, 2019.

[56] M. A. Kadampur and S. Al Riyaee, "Skin cancer detection: applying a deep learning based model driven architecture in the cloud for classifying dermal cell images," Informatics in Medicine Unlocked, vol. 18, p. 100282, 2020

[57] B. Vinay, P. J. Shah, V. Shekar, and H. Vanamala, "Detection of Melanoma using Deep Learning Techniques," in 2020 International Conference on Computation, Automation and Knowledge Management (ICCAKM), 2020: IEEE, pp. 391-394.

[58] F. Xiao and Q. Wu, "Visual saliency based global-local feature representation for skin cancer classification," IET Image Processing, vol. 14, no. 10, pp. 2140-2148, 2020.

[59] J. Daghrir, L. Tlig, M. Bouchouicha, and M. Sayadi, "Melanoma skin cancer detection using deep learning and classical machine learning techniques: A hybrid approach," in 2020 5th International Conference on Advanced Technologies for Signal and Image Processing (ATSIP), 2020: IEEE, pp. 1-5.

[60] S. Lu, Z. Lu, and Y.-D. Zhang, "Pathological brain detection based on AlexNet and transfer learning," Journal of computational science, vol. 30, pp. 41-47, 2019.

[61] Y. Zhai, H. Cao, W. Deng, J. Gan, V. Piuri, and J. Zeng, "BeautyNet: Joint multiscale CNN and transfer learning method for unconstrained facial beauty prediction," Computational intelligence and neuroscience, vol. 2019, 2019.

[62] M. A. Kassem, K. M. Hosny, and M. M. Fouad, "Skin lesions classification into eight classes for ISIC 2019 using deep convolutional neural network and transfer learning," IEEE Access, vol. 8, pp. 114822114832, 2020.

[63] A. Mahbod, G. Schaefer, I. Ellinger, R. Ecker, A. Pitiot, and C. Wang, "Fusing fine-tuned deep features for skin lesion classification," Computerized Medical Imaging and Graphics, vol. 71, pp. 19-29, 2019.

[64] T.-C. Pham, C.-M. Luong, M. Visani, and V.-D. Hoang, "Deep CNN and data augmentation for skin lesion classification," in Asian Conference on Intelligent Information and Database Systems, 2018: Springer, pp. 573-582.

[65] R. Ashraf et al., "Region-of-Interest Based Transfer Learning Assisted Framework for Skin Cancer Detection," IEEE Access, vol. 8, pp. 147858-147871, 2020.

[66] U.-O. Dorj, K.-K. Lee, J.-Y. Choi, and M. Lee, "The skin cancer classification using deep convolutional neural network," Multimedia Tools and Applications, vol. 77, no. 8, pp. 9909-9924, 2018.

[67] M. M. I. Rahi, F. T. Khan, M. T. Mahtab, A. A. Ullah, M. G. R. Alam, and M. A. Alam, "Detection Of Skin Cancer Using Deep Neural Networks," in 2019 IEEE Asia-Pacific Conference on Computer Science and Data Engineering (CSDE), 2019: IEEE, pp. 1-7.

[68] K. M. Hosny, M. A. Kassem, and M. M. Foaud, "Skin cancer classification using deep learning and transfer learning," in 2018 9th Cairo International Biomedical Engineering Conference (CIBEC), 2018: IEEE, pp. 90-93.

[69] D. Gavrilov, L. Lazarenko, and E. Zakirov, "AI Recognition in Skin Pathologies Detection," in 2019 International Conference on Artificial Intelligence: Applications and Innovations (IC-AIAI): IEEE, pp. 54542.

[70] B. Harangi, A. Baran, and A. Hajdu, "Assisted deep learning framework for multi-class skin lesion classification considering a binary classification support," Biomedical Signal Processing and Control, vol. 62, p. 102041, 2020.

[71] A. Demir, F. Yilmaz, and O. Kose, "Early detection of skin cancer using deep learning architectures: resnet-101 and inception-v3," in 2019 Medical Technologies Congress (TIPTEKNO), 2019: IEEE, pp. $1-4$.

[72] A. C. Salian, S. Vaze, P. Singh, G. N. Shaikh, S. Chapaneri, and D. Jayaswal, "Skin Lesion Classification using Deep Learning Architectures," in 2020 3rd International Conference on Communication System, Computing and IT Applications (CSCITA), 2020: IEEE, pp. 168-173.

[73] L. Wei, K. Ding, and H. Hu, "Automatic Skin Cancer Detection in Dermoscopy Images based on Ensemble Lightweight Deep Learning Network," IEEE Access, 2020.

[74] X. Li, J. Wu, E. Z. Chen, and H. Jiang, "From Deep Learning Towards Finding Skin Lesion Biomarkers," in 2019 41 st Annual International Conference of the IEEE Engineering in Medicine and Biology Society $(E M B C), 2019$ : IEEE, pp. 2797-2800.

[75] A. G. Pacheco, A.-R. Ali, and T. Trappenberg, "Skin cancer detection based on deep learning and entropy to detect outlier samples," arXiv preprint arXiv:1909.04525, 2019.

[76] B. Harangi, A. Baran, and A. Hajdu, "Classification of skin lesions using an ensemble of deep neural networks," in 2018 40th annual international conference of the IEEE engineering in medicine and biology society (EMBC), 2018: IEEE, pp. 2575-2578.

[77] I. Goodfellow et al., "Generative adversarial nets," in Advances in neural information processing systems, 2014, pp. 2672-2680.

[78] L. Lan et al., "Generative Adversarial Networks and Its Applications in Biomedical Informatics," Frontiers in Public Health, vol. 8, p. 164, 2020.

[79] I. S. Ali, M. F. Mohamed, and Y. B. Mahdy, "Data augmentation for skin lesion using self-attention based progressive generative adversarial network," arXiv preprint arXiv:1910.11960, 2019.

[80] H. Rashid, M. A. Tanveer, and H. A. Khan, "Skin Lesion Classification Using GAN based Data Augmentation," in 2019 41st Annual International Conference of the IEEE Engineering in Medicine and Biology Society (EMBC), 2019: IEEE, pp. 916-919. 
[81] P. Sedigh, R. Sadeghian, and M. T. Masouleh, "Generating Synthetic Medical Images by Using GAN to Improve CNN Performance in Skin Cancer Classification," in 2019 7th International Conference on Robotics and Mechatronics (ICRoM), 2019: IEEE, pp. 497-502.

[82] Z. Qin, Z. Liu, P. Zhu, and Y. Xue, "A GAN-based image synthesis method for skin lesion classification," Computer Methods and Programs in Biomedicine, p. 105568, 2020.

[83] M. B. Abdulrazzaq and J. N. Saeed, "A Comparison of Three Classification Algorithms for Handwritten Digit Recognition," in 2019 International Conference on Advanced Science and Engineering (ICOASE), 2019: IEEE, pp. 58-63.

[84] Z. Xu, F. R. Sheykhahmad, N. Ghadimi, and N. Razmjooy, "Computeraided diagnosis of skin cancer based on soft computing techniques," Open Medicine, vol. 15, no. 1, pp. 860-871, 2020.

[85] R. Sharma and M. Lal, "Skin cancer lesion classification using lbp based hybrid classifier," International Journal of Advanced Research in Computer Science, vol. 8, no. 7, 2017.

[86] A. A. Salih and M. B. Abdulrazaq, "Combining best features selection using three classifiers in intrusion detection system," in 2019 International Conference on Advanced Science and Engineering (ICOASE), 2019: IEEE, pp. 94-99. 